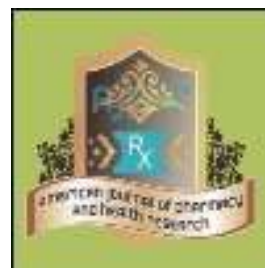

Review Article

AMERICAN JOURNAL OF PHARMACY AND HEALTH RESEARCH

www.ajphr.com

2019, Volume 7, Issue 2

ISSN: 2321-3647(online)

\title{
Irrational Use of Drugs - A Review
}

\author{
Assem Babbar, Ankit Sharma, Rahul Rawat, Naman Arora, Chirag Verma \\ SGRRITS, Patel Nagar, Dehradun-248001, Uttarakhand
}

\begin{abstract}
Everybody wants to get success in their life but on the cost of their health. Due to that the chances of irrationality of drugs increase in which self-medication is one of the major cause. The basic principle for the rational use of drug is better supply of drugs, better prescribing and lower costs for health care. When the basic principle of rational use of drugs is not fulfill it leads to irrationality which further leads to adverse effects and toxicity. There are number of factors which contributes to irrationality either they are inter-related to each other or they directly have affects and it may cause some effects on health and economic consequences. There is a cycle for rational use of medicine which help the clinician to prescribe the appropriate drug for appropriate disease condition, if each step is followed appropriately the irrationality improves. The concept of "rational use of medicine" can at times be confusing and not easily appreciated by patients, healthcare providers, policy makers, or the public, all of whom needs to collaborate effectively to address this challenge and the policy makers should be need to update with current trends.
\end{abstract}

Keywords: Irrational, Rational, Self-medication 


\section{INTRODUCTION}

In the current scenario, everybody wants to get success in their life but on the cost of their health. Due to that the chances of irrationality of drugs increase in which self - medication is one of the major cause. According to the WHO, the definition of rational use of drugs means "To fulfill the requirements of medications of the patient at right time, at right dose, at lower cost" But this definition is differ for consumers which is defined as "The rationality of using a drug is based on the interpretation of its value for daily life, which is influenced by the cultural perceptions and economic conditions". The basic principle for the rational use of drug is better supply of drugs, better prescribing and lower costs for health care ${ }^{2}$. When the basic principle of rational use of drugs is not fulfill it leads to irrationality which further leads to adverse effects and toxicity. According to the WHO, adverse effect is defined as "The unintended noxious effect which is occur at doses in man for prophylaxis, diagnosis or therapy in turns it may give such hazards effects for a fatal life and toxicity is defined as that a direct action of drug, often at high doses" $"$. There are some irrational combination of drugs which is used in market and leads to hazards effect like -

Norfloxacin + Metronidazole / Norfloxacin + Tinidazole.

This is the combination used in simple diarrhea. It is the combination of anti - amoebic used as anti-bacterial and both are broad spectrum but actually this combination may not required and it may cause resistance towards antibiotics ${ }^{3,2}$.

Domperidone + Rabeprazole / Domperidone + Esomeprazole.

This is the combination used in gastric problem but it may increase the chance of rhabdomyolysis ${ }^{3}$.

Fluconazole + Tinidazole / Doxycycline + Tinidazole / Tetracycline + Metronidazole

Combining two antimicrobial agents to increase the spectrum of activity which is irrational, as patient need only one $\mathrm{drug}^{3}$.

In addition to this, poly pharmacy and poor medication adherence also are the main culprits for causing treatment failure in patient which affects the efficacy of drugs and reach the sub therapeutic level which is also the one type of irrationality ${ }^{4,1}$.

There is criteria for rational use of drug, if the criteria is not fulfilled then the irrationality occurs. The criteria is as follows-

Appropriate indication - The drug which is used in therapy should be effective and safe ${ }^{1}$. Appropriate drug - In this the choice of drug is based upon its efficacy, safety and on cost ${ }^{1}$. 
Appropriate patient - In this right drug is given to the right patient, to minimize the adverse drug reactions and side effects ${ }^{1}$.

Appropriate patient information - In this the information about the patient should be clear like past medication history, surgical history ${ }^{1}$.

Appropriate evaluation - The effect of the medication should be monitored thoroughly and appropriately ${ }^{1}$.

\section{Reasons of Irrationality}

There are the number of causes which participates in it but some plays the pioneering role which is described as follows-

Irrational prescribing - It is the cause which is difficult to cure but it is on the top most position in the medical field for not following the rationality ${ }^{1,3,9,10}$. The irrationality in irrational prescribing occurs due to number of reasons which are as follows-

Inappropriate use of drugs where there is no need

For example- the use antibiotics in case of viral upper respiratory infections.

1. Usage of wrong drugs in different conditions which require specific drug therapy. For example - use of tetracycline in childhood diarrhea which require ORS therapy

2. When there is wrong drug administration or duration error occurs.

For example - use of IV metronidazole where the oral metronidazole works appropriately

3. When the drug unable to provide require efficacy\& bio - availability like unable to reach therapeutic level it also leads to irrationality.

\section{Over use / Over consumption}

It is the one of leading cause in developing countries especially in India which cause irrationality. Even though, the consumers and prescribers expectation mismatches due to that the over consumption of medicines which also cause irrationality, especially occurs in the case of antibiotics use. Owing to it, prescribing and dispensing patterns are influenced by socio-cultural factors such as patient demand, prescribing attitude to risk, previous prescribing experience and drug promotion ${ }^{1,10,11}$. The other examples of this reason is are following belowAnti - diarrhoeals, pain killers, cough preparations etc ${ }^{1}$.

\section{Multiple drug use or poly pharmacy}

It is the noteworthy problem of both that is of prescriber as well as consumer like it acts as a fire on each side. In the case of prescriber, the prescriber can prescribe more number of drugs either it is required or not which not only affects the health of the patients even it imbalance the its budget also and in case of consumers, the consumer like to buy multiple drugs from private or 
informal sector which leads to hazards effects on their health. On every side of coin, it leads to irrationality ${ }^{1,4,10,11}$.

\section{Absence / weak of national drug policy}

If the national drug policy is not implemented properly, it may become obstacle. If it is implemented properly then there is proper implementation of drug regulation, adequate storage facilities and good distribution takes place ${ }^{1,10}$.

\section{Faculty and inadequate training and education of medical graduates}

It includes the lack of clinical training in the clinicians which can lead to irrational use of $\operatorname{drug}^{3,10}$. It includes -

1. Improper writing of the clinician ${ }^{7,10}$.

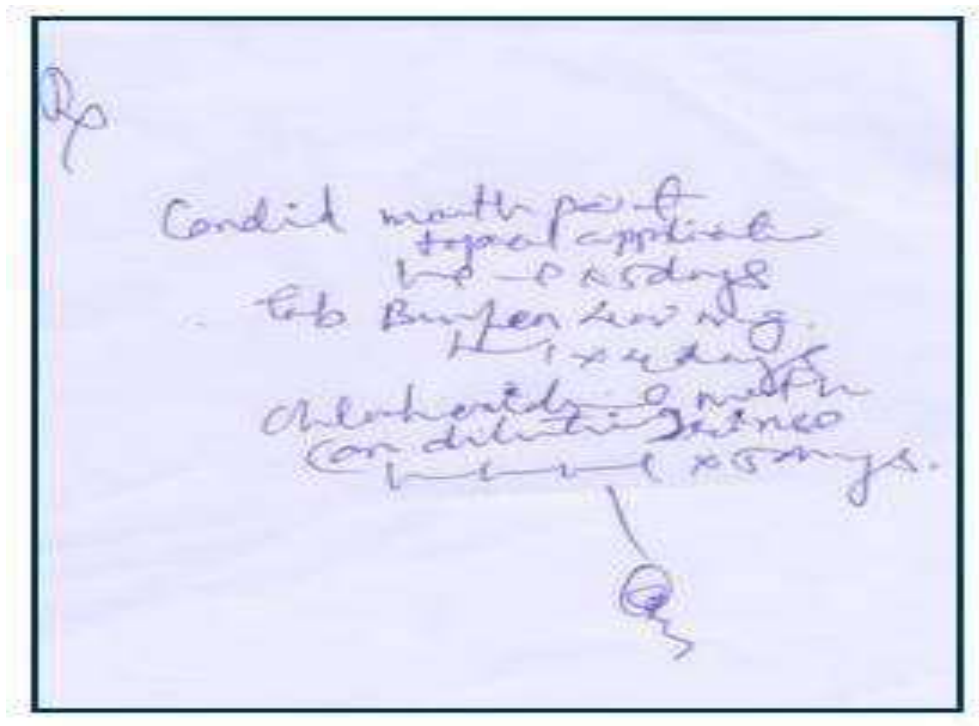

2. Lack of clinical diagnosis skill ${ }^{3,10}$.

Poor communication between health professionals and patients - In this scenario, there is a lack of communication between patient and clinician because nobody have mean time to kill on their health for their welfare either due to increase in work load on both sides or due to any other reason which is not a good indication for rational use of $\mathrm{drug}^{3,10}$.

Demand of patient - It is the duty of the clinician to fulfill the demand of the patient, for the sake of patient the prescriber prescribe the medication for each complaint of patient which is not necessarily in all the cases and it precedes to poly-pharmacy indirectly which further sparks irrational use of $\operatorname{drugs}^{3,4,10}$.

Lack of Information - It is the factor in which every person is involved and it starts from physicians, then pharmacists, nurses and in the end the patient itself. Like that, sometimes the physician does not familiar or having insufficient knowledge about new drugs/ combinations \& 
pharmacist also don't have sufficient knowledge about the drugs to dispense, nurses also don't know about the drugs to administer and similarly patient also don't know about the drugs they consume. Due to these reasons they all fall in this category ${ }^{6,10}$.

There is a quote on that "Doctors pour drugs of which they know little, To cure diseases of which they know less into patients to whom they know nothing".

\section{Types of Irrational Use Of Drugs}

There are different types of irrational prescribing which is considered as types of irrational use of drugs. It represents as follows-

Under - Prescribing - It is that type of cause which generally occurs in the hospitals of developing countries ${ }^{5}$. However, it mostly occurs in two type of cases-

1. Needed medication is not prescribed ${ }^{5}$.

2. The dosage prescribed is inadequate ${ }^{5}$.

Over - Prescribing - Owing to this type, it generally occurs in the cases of-

1. The prescribed drugs is not needed by the patient ${ }^{5}$.

2. The quantity of the drug dispensed too much for the current course of treatment ${ }^{5}$.

Incorrect Prescribing or Dispensing - Despite the above types of irrationality, it is the type which leads to number of errors which indirectly or directly affect the rational use of $\operatorname{drug}^{5}$. It happens when-

1. When prescribing the wrong drug 5 .

2. Dispensing the wrong drug when the prescription is not prepared properly ${ }^{5}$.

3. Adjustments are not done for existing medical, genetic or environmental conditions ${ }^{5}$.

Extravagant Prescribing -Although this the type which does not follow the pattern of rational use of drug but in this type the different type of things participate which give them more encouragement for this such as-

1. When the less expensive generic drugs are available then why the more expensive branded drugs are prescribing ${ }^{5}$.

2. Treating the patients according to their symptoms instead of treating his/ her serious illness which not affect their health even the treatment cost increases ${ }^{5}$.

Multiple Prescribing - It is the type which is similar to poly - pharmacy like when the fewer drugs produce same effect rather than prescribing number of medications ${ }^{5}$.

\section{Factors Underlying Irrational Use Of Drug}

There are number of factors which contributes to irrationality either they are inter-related to each other or they directly affects. It includes- 


\section{Health system}

The health system is the factor which indirectly leads to irrationality because the health system is itself affected by some reasons that is drug shortage, improper supply of drugs, expired drugs etc. due to that the patient as well as prescriber feels dwindling in the confidence as well as it gets mandatory for the prescriber to prescribe the available drug only even he / she knows that it is not correct medicine to treat that condition ${ }^{5,9,10}$.

\section{Prescriber}

It is the factor which is affected by both external and internal factors. He or she made error either due to inappropriate handwriting, lack of adequate knowledge and practice, limited knowledge or experience without having recent updates, due to pressure of heavy workload or insufficient staff. Even though, it may occurs due to lack of drug information or due to unable to make clear diagnosis. If the cause is lack of drug information, then the prescriber have limited choice for the drug selection. In addition to limited choice even the ADRs may occur which is itself an indicator of medication misuse due to poor prescribing practices and lack of compliance. The number of factors which participate in ADRs are co - morbidity, polypharmacy, older age etc $^{5,9,10}$.

\section{Dispenser}

The drug dispenser plays pioneering role in the rational use of drug which ultimately affect the therapeutic effect. The quality of dispensing together with knowledge about drugs affect the whole process like if there is shortage of products, it indicates that there is lack of supervision. Besides this, if there is shortage of dispensing time due to heavy patient load or any other reason it may also cause irrationality which ultimately produce adverse effect on the patient health ${ }^{5,9,10}$.

\section{Patient and Community}

It is the factor which is inter - related to each other because of their cultural beliefs, their communication skills, attitude towards prescriber ,limited time available for consulting, community believes towards drug efficacy or towards route of administration, heavy cost of treatment which affects the rationality of drug use. It is the one of leading cause for irrationality $2,5,9,10,11$.

\section{Drug Explosion}

It means that the explosion of drugs that is infinite number of drugs which is used by the prescriber which may complicated the choice of appropriate drug for particular indication ${ }^{3,5,9,10}$.

\section{Consumer Protection Act}


The consumer protection act in medical profession may restrict the clinician regarding prescription which itself leads to irrationality ${ }^{3}$. In the consumer protection, it is mention that don't use substitute but when there is emergency or drug is not available then sometimes the substitutes are used. From that it is crystal clear that itself consumer protection restrict the doctors to prescribe drugs $3,5,9,10$.

\section{Impacts of Irrational Use of Drugs}

There are number of irrationality occurs but it may cause some effects on health and economic consequences. The health related impacts may be quantified rarely, because it is possible in some drugs only. The following are the impacts which is produced by inappropriate use of drugs:-

\section{Adverse and Possible lethal effects}

In this impact self - medication scores higher number which may cause these effects but it also occurs due to the mistake of the clinician like in the case of anti-biotic misuse or overuse. These are some examples which leads to these type of effects ${ }^{1,2,11}$.

\section{Limited Efficacy}

It is the cause which usually occurs when the dose is under therapeutic range or the dosage is not suitable according to the patient condition ${ }^{1,2,11}$.For example : therapeutic dosage of tuberculosis drugs or leprosy drugs.

It is the condition where the drug don't achieve therapeutic window and the sub- therapeutic level is not able to produce desired pharmacological effect.

Risk of Infection - It is the leading cause of death in India and other countries, thus this type of irrationality of drugs may lead to death, decrease the life of the patients or it may lead to life threating diseases such as HIV, AIDS etc. The basic reason for this outcome is improper use of injections ${ }^{7,11}$.

\section{Morbidity and Mortality}

The improper use of drugs will increase the chance of morbidity and mortality and it occurs due to number of reasons for instance self medication, antibiotic resistance, drug allergy, poly pharmacy, co-morbidity of diseases etc. It is the one of biggest health hazard occur in the patients and it is non-reversible type of end result which further lead to reduced quality of therapy $^{3,7,11}$.

\section{Wastage of the Resources}

There is scarce of resources occurs due to irrationality of drugs, when the drugs are used unnecessarily which is not required it leads to increase the hospital stay and treatment $\operatorname{cost}^{7}$.

\section{Psychological effect}


It is the indiscriminate type of effect which is due to increase dependency of drugs when the drugs are prescribed irrationally. It mostly occurs in the case of narcotics. There is one quote which suits “A PILL FOR EVERY ILL” which admires that excessive demand of medicines ${ }^{7}$.

\section{Clinical toxicity}

The clinical toxicity occurs commonly in the hospital either due to the drugs which is used in daily life like as Acetaminophen or the drugs in special condition of the patient like as Isoniazide in tuberculosis patient. The both types of the drugs shows direct action which depends upon the dose of that drug which further damages the organs of the body $\mathrm{y}^{3,7,11}$.

For example- Over Dose of Acetaminophen may cause liver damage.

High Dose of Isoniazide may cause peripheral neuropathy.

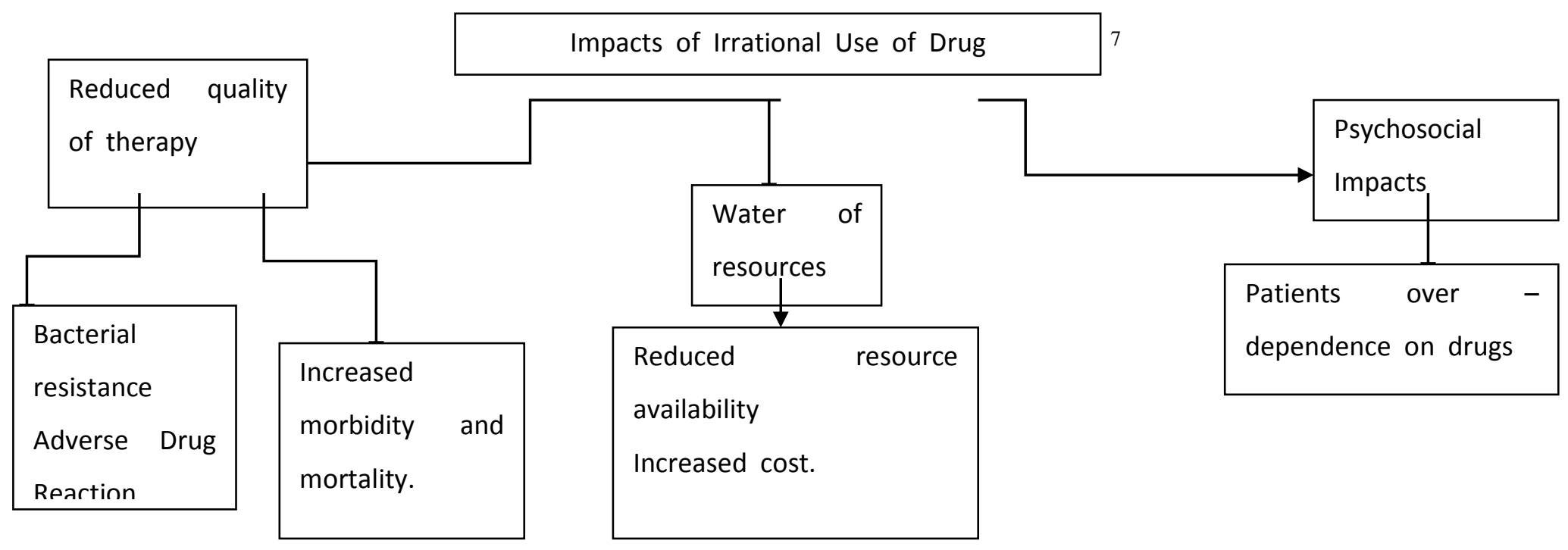

\section{How to improve irrationality?}

Cycle of Medicine Use-

There is a cycle for rational use of medicine which help the clinician to prescribe the appropriate drug for appropriate disease condition and it is the first step as well as rate limiting step of the cycle. Besides this, there are further three steps which is well performed by the other health care professionals and which helps to complete the cycle of medicine use. If each step is followed appropriately the irrationality improves and the last step is to monitor the patient and treatment for better outcome of the patient.

There are the four steps in the cycle which are as follows - 


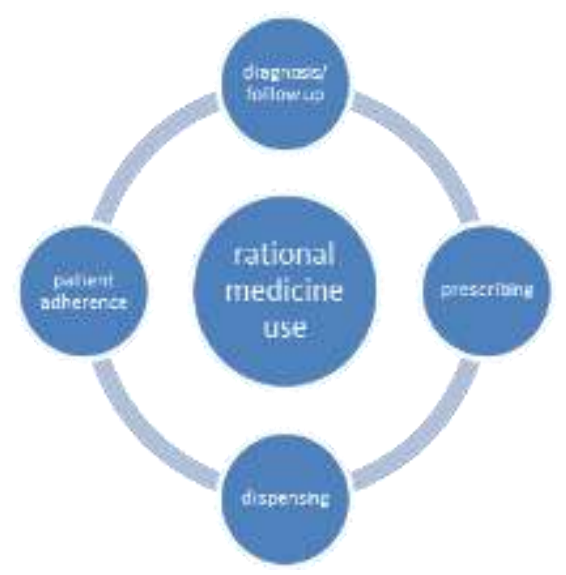

\section{Diagnosis / Follow up}

The diagnosis stage involves identifying and defining the problem(s) which require intervention. This initial stage can set up a cycle of inappropriate medicine usage if the wrong problem is diagnosed by the clinician for the intervention. If in the case of wrong intervention is outlined the patient will get wrong medicines which further interrupts the cycle and it leads to irrationality ${ }^{3,8}$.

\section{Prescribing}

Appropriate Indication - the decision to prescribe medicine (s) is entirely depends on the drug therapy which should be

Appropriate drug - the
selection of drugs depends upon
its efficacy, safety, suitability
and cost considerations.

Appropriate patient - the drug is accepted to the patient if there is no contraindications exists or minimal adverse drug reactions

$$
\begin{aligned}
& \text { Appropriate monitoring - the anticipated and } \\
& \text { expected effects of medicines should be } \\
& \text { appropriately monitored. }
\end{aligned}
$$

It is a complex process - one which tests the healthcare providers knowledge or application of therapeutic principles, communication skills, as well as their approach to uncertainties. Prescribing process depends upon the goal of the therapy which is influenced by the patient expectations and preferences. Prescriber confront the task of choosing from many options but the final pharmacological choice depends upon the benefit - risk analysis of medicine, patient factor, its availability and cost. Patient factors that may influence the medicine selection process include physiological status(pregnancy, kidney failure),drug- drug interactions, adverse reactions, drug factors like pharmacokinetic and pharmacodynamic. To balance the irrationality in this step 
which includes over prescribing, under prescribing, incorrect prescribing, extravagant prescribing, multiple prescribing the prescriber should become a good prescriber by achieving four aims in their mind- maximize effectiveness, minimize risks, minimize costs, respect patient's choice ${ }^{3,8}$. According to the WHO there are five requirements outlined for the prescriber for rational medicine use-

\section{Dispensing:}

The dispensing is the stage in which the patients receive their medications and often dispensing is carried out by the pharmacist and the technicians. In the clinical practice, the prescribing and dispensing activities is considered to be a safety mechanism which assure independent assessment of the proposed therapy before patient begins treatment. For instance if the dispensing practices such as counting, labeling, packaging is poorly executed, they are likely to impact the patient's confidence in the dispensed products and which subsequently compliance to therapy as well as cause irrationality of the drugs. According to the WHO, some necessary actions should be taken by the dispenser to minimize the irrationality by cross -checking the prescription, review the legal requirements, safety and appropriateness ${ }^{3,8}$.

\section{Patient - adherence:}

The engagement between dispenser and the patient is also key, as it can significantly impact how the medicines are used by the patients. For the instance, adherence is likely to improve only if the patient understands the importance of taking the medications, can follow the instructions correctly and appreciates the risks of non - adherence ${ }^{3,8}$.

\section{Monitor the treatment as well as the patient, so that the patient should get better outcome. CONCLUSION}

After evaluation it is concluded that irrationality is a major global health challenge with significant implications for patients, health care systems and communities. Such practices are likely to lead to poor health delivery that may put patients at risk and result in wastage of scarce resources that could have been used to tackle other pressing health needs. The concept of "rational use of medicine" can at times be confusing and not easily appreciated by patients, healthcare providers, policy makers, or the public, all of whom need to collaborate effectively to address this challenge and the policy makers should be need to update with current trends.

\section{REFERENCES}

1. Grand Le Amanda, Hogerzeil V Hans, Ruskanp Haaizer Feor “Intervention Research In Rational Use of Drugs - Review”, Health Policy \& Planning, Oxford University Press 
$1999,14(2), 89-102$.

2. Shivhare S.C, Kunjwani H.K etal "Drug Hazards \& Rational Use of Drugs - A Review", Journal of Chemical \& Pharmaceutical research, 2010, 2 (1); 106 - 112.

3. Ambwani Dr. Sneha, Mathur Dr. AK "Health Administration vol; XIX number 1 : 5-7, Chapter - 2, Rational Drug Use.

4. P Shree Divya, Prosanna Lakshmi et al, "Evaluation of Knowledge, Attitude \& Practice Towards Drug Use along with Good Pharmacy Practice among Pharmacy Students", International Journal of Pharmacy, ISSN - 2230 - 8407, 2017 ; 8 (3).

5. Alfan \& Nesta Ferguson Trust, Distance Education Programme "Drug Management \& Rational Use , Unit - 5", African Medical Research Foundation 2007.

6. Mohanta G. P, Kumar Vijaya I, Manma P. K, Manava R, "Independent Unbiased Drug Information A Need To Promote Rational Drug Use", Health Administer vol: XX Number 1\&2: 99 - $10 \mathrm{Pg}$.

7. Panchbhai S Dr. Arati, M.D.S, Department of Oral Medicine \& Radiology, SP Dental College \& Hospital, DMIMS University ( Maharashtra), "Rationality Of Prescription Writing”, 2014 Janurary 21.

8. Aseno Ofori - Richard, Agyeman Adom Akosna "Irrational Use of Medicine - A Summary of Key Concepts", Research Unit, Health Policy, 2016 October 28.

9. Porter Gillian, Grills Nathan "Medication Misuse In India: A Major Health Issue In India”, Journal of Public Health, 2015 June 8: vol 38,no.2,pp e150-e157.

10. Calik Esra, Sencan Nagli M, Philip M "Factors Affecting Rational Drug Use, Compliance \& Wastage”, Turk J. Pharm Sci, 2013, 10(1), 15-170.

11. Okyay Azim Ramazan, Erdogan Aysegul "Self- Medication Practices \& Rational Drug Use Habits Among University Students - A Cross Sectional Study From Kahramanmaras, Turkey, Peer J Journal, 2017 November 1, DOI: 10.7717/peerj.3990. 